\title{
A Design Entropy Based Hybrid Soft Classifier Algorithms for Improving Classification Performance of a Satellite Data
}

\author{
Ranjana Sharma $^{1 *}$, P. K. Garg ${ }^{1}$ and R. K. Dwivedi ${ }^{2}$ \\ ${ }^{1}$ Professor, IIT Rorkee, India \\ ${ }^{2}$ Prof \& Principal, CCSIT, Teerthnakar Mahaveer University, Moradabad, India \\ ${ }^{*}$ Corresponding author
}

\begin{abstract}
Article Info
Keywords: Accuracy, Entropy, Fuzzy C-Means with entropy, Mixed classification/Soft classification, Pure classification.

2010 AMS: 00A69

Received: 13 September 2018

Accepted: 28 January 2019

Available online: 30 August 2019
\end{abstract}

\begin{abstract}
Image classification of the satellite imagery interprets the thematic map to represent the spatial distribution of earth features. There are so many applications of Remote sensing image classification such as Resource utilization and environmental impact analysis etc. The overall process result depends on two main aspects Every object have distinctive signature and feature of interest The process can distinguish these features separately. Image classification is broadly classified in two ways Pure classification and Mixed classification. In the pure classification, the pixels are classified into class only and in the mixed classification, pixels can fit into one or more module according to their membership values. In hard classification, data may be lost because of the restriction being in a single class. However in the soft classification, this problem is resolved. After resolving the problem, there is a need of accuracy assessment. Accuracy parameter is very important factor in terms of classification. So, in this study, I am trying to design the algorithm for the hybridization classification with entropy to maintain the optimizing.
\end{abstract}

\section{Introduction}

Usually the pure classification is used to generate thematic maps and surface information. The pixels of the pure classification give particular land cover information. Conversely this defeat the real data of geographic surface, the covered data might be present in more than one land. The mixed pixel likelihood is higher for course resolution. The data in satellite is mixed and it gives numerous surface of earth information within pixel that is a big problem in terms of categorization or in accuracy. Accuracy assessment is again an issue in soft classification. Researchers and analyst have made great hard work in budding advanced classification approaches[26,28] for optimizing categorization appropriateness $[13,8,26,29]$.Predictable methods of correctness measurement mandatory harden the surface of earth information. This once again leads to an inaccurate estimation. In remote sensing, both supervised and unsupervised classification techniques may be applied to perform soft classification [1]. It increases the classification accuracy and produces adequate land cover composition. Digital image categorization is typically depending upon to retrieve spectral information using a range of statistical classification techniques such as Maximum Likelihood Classifier (MLC), k-means clustering, Minimum Distance to mean classifier etc. The allocation of each pixel of the data from these classifiers in a single class thus produces pure or hard classification. However, often the pixels of the satellite do not represent a single class but contains more classes in single pixel area[30]. This situation is quite prevalent in developing country like India where the development has taken place in a haphazard manner. In a satellite image having coarse resolution, chances of class mixture within a single pixel are higher in the heterogeneous landscapes, and in interclass boundaries leading to higher proportion of mixed pixels in an image. Fine resolution satellite data, will be able to remove, by and large, the mixing of information within a pixel, yet the problem may still exist at inter-class boundary, where the number of such pixels may increase many folds. Whatever be the origin of mixed pixels, these may generate problem in image categorization. For instance, a miscellaneous pixel shows a combined spectral response that may be unlike to the spectral response of each of its component classes and, therefore, the pixel of satellite may not be allocated to any of its division classes [39]. Hence, error may occur in the classification of image [9] containing large number of mixed pixels. Hard classification approaches,

Email addresses and ORCID numbers: pal.computers@tmu.ac.in (R. K. Dwivedi)

sharmaranjana04@gmail.com, 0000-0002-0088-715 (R. Sharma), pkgiitr@gmail.com (P. K. Garg), princi- 
can work only on single class but mixed pixels to be allocated more than one class. So information of pixel of image loss and we can say hard classification have only single class information. In case of hard classification methods mixed pixels may thus be treated as error or uncertainty, or uncertainty in class allocation. The land use land cover areal estimate obtain from hard classification, if used as an input to any Geographical Information System (GIS) based application, it may affect the accuracy of the end product. Thus, mixed pixels of image are not to be handed by hard classification. The problem of mixed pixels may be resolved by accommodating this in the classification process in some way to acquire the hidden information[14,24].The application of soft classification methods based on spectral mixture analysis[20],fuzzy set theory[21] may thus be adopted. The output from these methods is a set of class membership values for each[8], pixel, also named as soft or fuzzy classification outputs, which are represented as probability, fraction or proportion images [30]. The utilization of soft classification methods is an active area of research, which can be gauged from a number of research papers published during the last couple of years[11,23,25,30]. Hybrid soft classification methods are largely in their exploratory stage. The research needs to be conducted to examine these methods on different remote sensing data products acquired in complex and uncertain environments.

\section{The Types of Entropy Based Hybrid Soft Classifiers}

The measure of information, as per Shannon [32,33] states that it has an intimate relationship with entropy theory as in statistical thermodynamics. Therefore, information theory and thermodynamics must have some common points of interest. The increase in entropy has been regarded as the degradation of energy by Kelvin[16]. In statistical thermodynamics, entropy is defined as a measure of the disorder of a system. However, in information theory, entropy[10] is a measure of the lack of information about the actual structure of the system [19]. It is perceived that fuzzy based information can become complete by adding entropy to the standard one, since it can observe the nature of both methods more deeply by contrasting these two methods[4,7]. In this study, it has been observed that entropy based method is similar to a statistical model having Gaussian distribution, since both of them have error functions, while the standard method such as FCM[15], PCM[17], etc. are different from a statistical model. For this reason, standard method is purely fuzzy, while entropy based method connects a statistical model and a fuzzy model[3,4,7]. In this study, one of the primary motivations is to hybridize FCM and PCM with entropy FUZZY C-MEAN WITH ENTROPY (FCMWE) CLASSIFIER Fuzzy c-Mean with Entropy (FCMWE) is the hybridization approach of classification where the emphasis is to integrate entropy based regularization method with FCM [12,15]. It is believed that the methods of Fuzzy c-Means become complete by adding entropy to the standard one as defined in Eq. (2.1)[7,19,22].

i) This hybridization has been proposed, to evaluate the Performance of algorithm which is entirely fuzzy, even as entropy based method is more similar to the statistical method.

ii) The principle of this process is based upon maximum entropy[31] which is further advance in various applications.

iii)[4][7] focus on comparison between methods and explain the entropy based algorithm more efficiently.

It is observed that the method of [3][7], also known as the standard method of FCM, is purely fuzzy, while entropy-based method is more similar to statistical models 34],[35]-[38]. The result which obtains the soft classifier gives result with higher uncertainty. But hybrid based classifier with optimum regularizing parameter generates classified output with lowest amount of uncertainty [18]. As nonlinearity, introduced by [7] and [4], smoothens the crisp solution into a differentiable one. Moreover, fuzzy solution approximates the crisp one i.e., the fuzzy solution converges to a crisp solution as m approaches to 1 .

\subsection{Fuzzy c-Mean with Entropy (fcmwe) Classifier}

Fuzzy c-Mean introduces non-linearity using $\left(\mathrm{u}_{k i}\right) \mathrm{m}$. However, use of entropy is another type of nonlinearity. The process of regularization is completed by adding a new function which known as regularizing function. The basic objective function of FCM with entropy classifier and flowchart are given in Eq. (2.1) and Fig.(2.1)

$$
J_{F C M W E}(U, V)=\sum_{i=1}^{c} \sum_{k=1}^{n} u_{k i} D\left(x_{k}, v_{i}\right)+v \sum_{i=1}^{c} \sum_{k=1}^{n} u_{k i} \log u_{k i},(v>0)
$$

Where $\mathrm{v}$ is regularizing parameter and has a value greater than 0. In the Eq. (2.1), the first term is the objective function of FCM classifier and second term is a nonlinear regularizing entropy function. It is observed that regularizing function is a strictly a convex function, and hence capable of fuzzifying the membership values.

\subsection{Possibilstic c-Mean with Entropy (pcmwe) Classifiers}

PCM c-Mean with Entropy (PCMWE) is a hybridization approach of classification where the emphasis is regularizing term. The working of algorithm gas defined in Fig. (2.2). The assigns pixel of image can vary some time its belong single cluster and some its belong more than one cluster. We know the membership of pixel does not follow the limit in FCM called hyper-line constraint. The entropy methods are support to rediscover repeatedly in fuzzy Clustering by different formulations. This hybridization has been proposed, to evaluate the Performance of algorithm which is purely fuzzy, while entropy based method is more similar to statistical method. The FCME and PCME clustering algorithm are nature in iterative where membership value are obtained by minimizing the generalized least- square error objective function[17], is obtained by minimizing objective function as,

$$
j_{m}(U, V)=\sum_{i=1}^{N} \sum_{j=1}^{C}\left(\mu_{i j}\right)^{m}\left\|x_{i}-v_{j}\right\|_{A}^{2}+\sum_{i=1}^{c}\left(1-\mu_{i j}\right)^{m}+v\left(-\sum_{i=1}^{C} \mu_{i j} \log _{2}\left(\mu_{i j}\right)\right)
$$

Where $\mathrm{v}$ is regularizing parameter and has a value greater than 0 in Eq. (2.2).

\section{The Study Area of Research}

Study area of research Sitarganj Tehsil which is situated close to Pantnagar, Uttarakhand State,India which is shown in Fig. (3.1). The survey is based on remote sensing data from IRS P6, AWIFS, LISS-III and LISS-IV sensors. and the The on-board sensors on this satellite are 


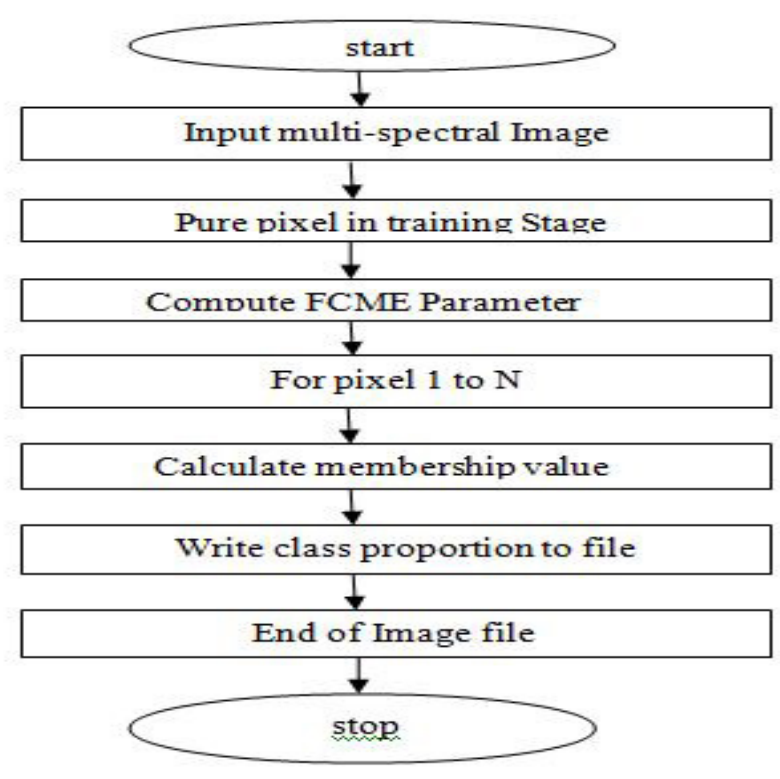

Figure 2.1: Flow chart of FCME classifier in supervised mode

LISS-IV (Linear Imaging Self Scanner), LISS-III and AWiFS.Fig. (3.1) describes these sensors characteristics in details.

\begin{tabular}{|l|l|l|l|l|}
\hline Sensor & Bands & Resolution [m] & Swath [km] & $\begin{array}{l}\text { Quantization } \\
{[\text { bits] }}\end{array}$ \\
\hline $\begin{array}{l}\text { LISS-IV Mono } \\
\text { mode }\end{array}$ & red & 5.8 & 70.3 & 7 \\
\hline $\begin{array}{l}\text { LISS-IV MX } \\
\text { mode }\end{array}$ & green red NIR & 5.8 & 70.3 & 7 \\
\hline LISS-III & $\begin{array}{l}\text { green red NIR } \\
\text { SWIR }\end{array}$ & 23 & 141 & 10 \\
\hline AWifS & $\begin{array}{l}\text { green red NIR } \\
\text { SWIR }\end{array}$ & $\begin{array}{l}56 \text { (nadir) } \ldots \\
70 \text { (edge) }\end{array}$ & 740 & 10 \\
\hline
\end{tabular}

Table 1: Resourcesat-1 payload characteristics

\section{Methodology of Research}

Uncertainty reduction or noise process by[6] for soft classification required classified fraction images and reference classified fraction images (if available), perform sampling over classified data, apply accuracy assessment method on sampled data and finally produces accuracy [6] parameters. Accuracy assessment method[5][16] of sub-pixel categorization is also Conrad data, such as SCM, FERM, SCM, error matrix, RMSE and Entropy. It is known that these methods required reference data accept entropy. But Entropy is does not require any reference data , so it knows as absolute indicator. Accuracy assessment process for soft classification required classified fraction images and reference classified fraction images (if available), perform sampling over classified data, apply accuracy assessment method on sampled.

\section{Result and analysis and discussion}

In this study, Fuzzy C-means with Entropy(FCME)and PCM c-Mean with Entropy (PCMWE) have been used as a base soft classifier and entropy has been added to investigate the effect of this hybridized model known as FCMWE and PCMWE. The basic objective of this study is to identify the optimized value of regularizing parameter $v$ for FCMWE classifier and PCMWE classifier which generates classified output with minimum uncertainty. To obtain accurate information from this classifier, the optimization of regularizing parameter $v$ is required. To perform the FCMWE, PCMWE classification, fixed value of $\mathrm{m}=1$ has been used for all varying values of $v$ (from 0 to $10^{9}$ ). The class membership $\mu$ increases till $v=10,10^{2}$ and $10^{3}$, the class membership is higher and lies between 0.91 to 0.99 for all the six classes. Regularizing parameter $v$ is the fixed parameter, $0 \leq v<\infty$ which regularizes the fuzzified solution to crisp solution. and thereafter it starts to decrease or becomes almost constant. \{Fig.(5.1), Fig. (5.2) and Fig. (5.3) $\}$

Thus, as per the analysis of class membership, the optimum value of $v$ for FCMWE classifier has been fixed as 105 . However, this optimization would be further verified by entropy. 


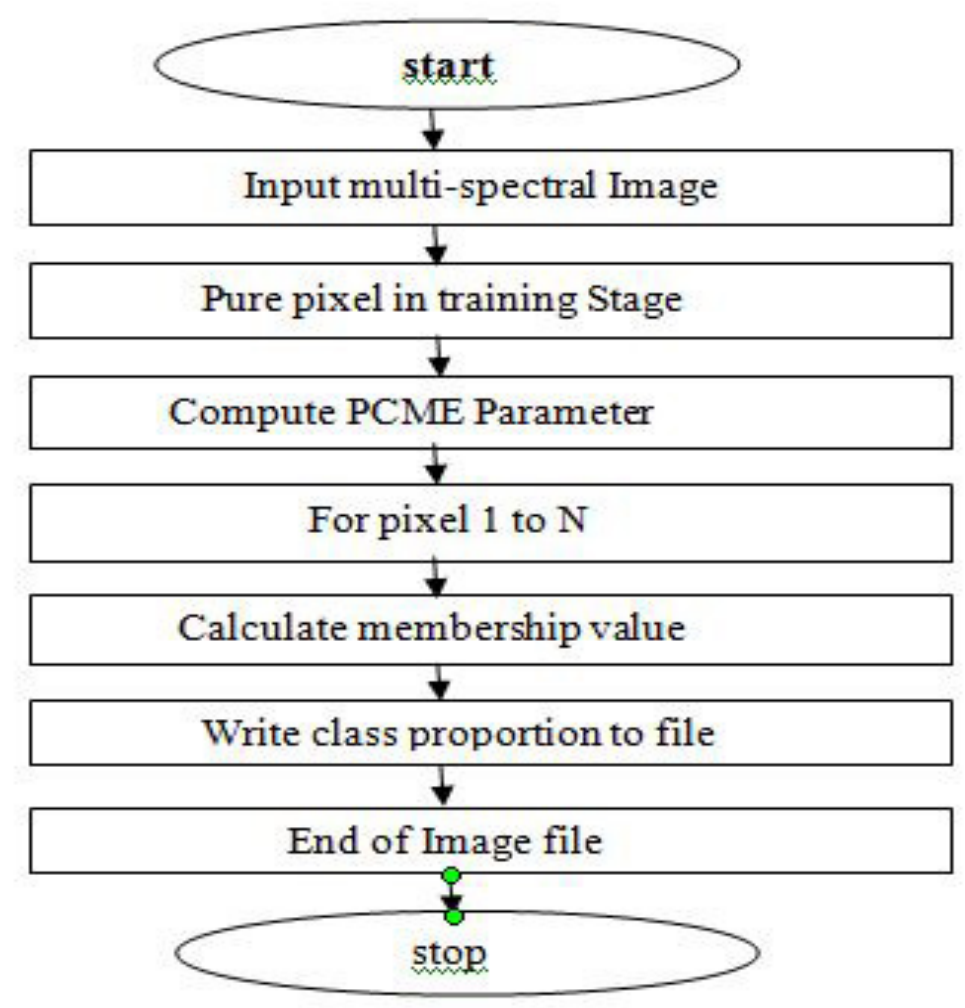

Figure 2.2: Flow chart of PCME classifier in supervised mode

\subsection{Calculate Entropy of hybridize classifier (fcme)}

The entropy of FCMWE classifier of classified fraction images can be computed by using Eq. (2.1) Fig. (5.1), Fig. (5.2) and Fig. (5.3) shows the computed entropy for AWiFS, LISS-III and LISS-IV fraction images of FCMWE classifier. It has been observed from Fig. (5.4), Fig. (5.5) and Fig. (5.6) that for $v=10^{2}$ and $10^{3}$ the entropy values for all classes are low. For this optimized value of $v$, the membership is high i.e. up to 0.996 and the computed entropy is low 0.004 .

This trend reflects that the uncertainty in results is low. In a nutshell, it can be concluded that whenever entropy has been used as an indirect accuracy measure and this shows the classification consistency with respect to a particular class.

\begin{tabular}{|l|l|l|l|l|l|l|l|}
\hline & \multicolumn{3}{|l|}{ Class membership } & \multicolumn{2}{l|}{ entropy } & $\begin{array}{l}\text { Optimized Mean } \\
\text { value }\end{array}$ \\
\hline & AWiFS & $\begin{array}{l}\text { LISS- } \\
\text { III }\end{array}$ & LISS-IV & AWiFS & $\begin{array}{l}\text { LISS- } \\
\text { III }\end{array}$ & LISS-IV & \\
\hline Agriculture & $10^{3}$ & $10^{3}$ & $10^{3}$ & $10^{2}$ & $10^{3}$ & $10^{2}$ & $7 \times 10^{2}$ \\
\hline Bright Forest & $10^{3}$ & $10^{3}$ & $10^{3}$ & $10^{3}$ & $10^{2}$ & $10^{2}$ & $7 \times 10^{2}$ \\
\hline Dense Forest & $10^{3}$ & $10^{3}$ & $10^{2}$ & $10^{2}$ & $10^{2}$ & $10^{2}$ & $4 \times 10^{2}$ \\
\hline $\begin{array}{l}\text { Agriculture Dry } \\
\text { land }\end{array}$ & $10^{2}$ & $10^{2}$ & $10^{2}$ & $10^{2}$ & $10^{2}$ & $10^{2}$ & $10^{2}$ \\
\hline $\begin{array}{l}\text { Agriculture Moist } \\
\text { land }\end{array}$ & $10^{2}$ & $10^{2}$ & $10^{2}$ & $10^{2}$ & $10^{2}$ & $10^{2}$ & $10^{2}$ \\
\hline Water Body & $10^{2}$ & $10^{2}$ & $10^{2}$ & $10^{2}$ & $10^{2}$ & $10^{2}$ & $10^{2}$ \\
\hline
\end{tabular}

Table 2: Class wise parameter optimization of(v) for FCME classifiers 


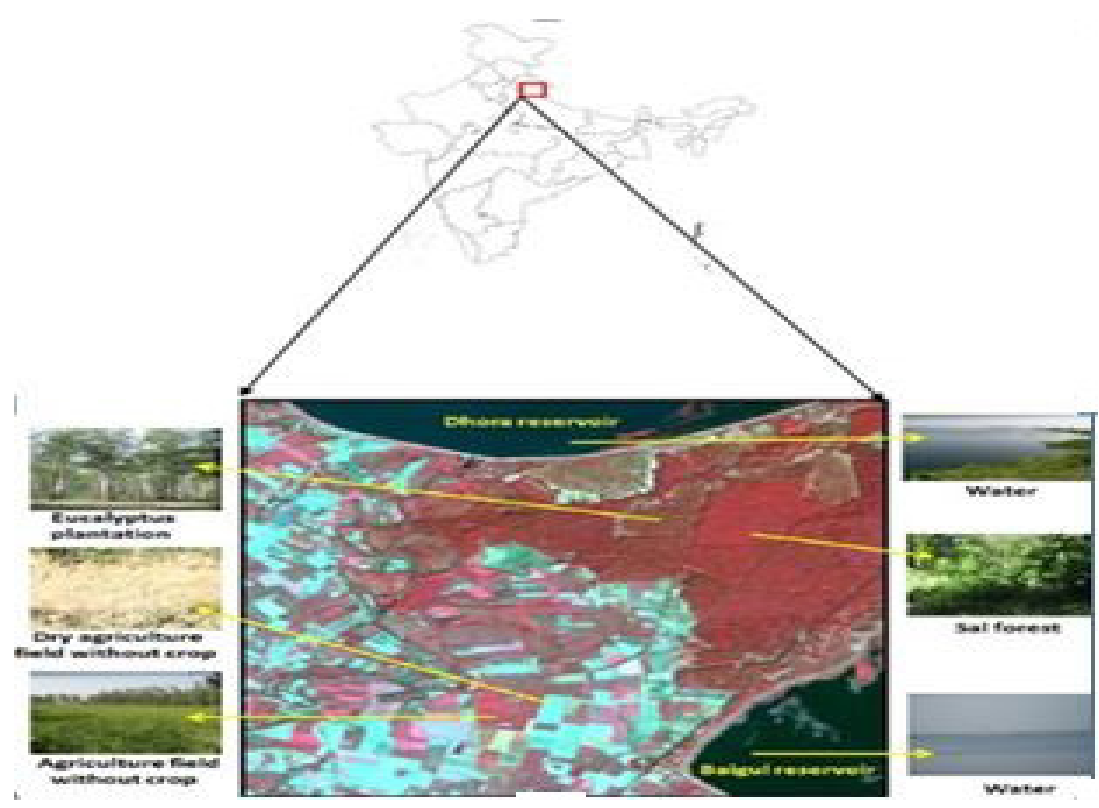

Figure 3.1: Location of study area

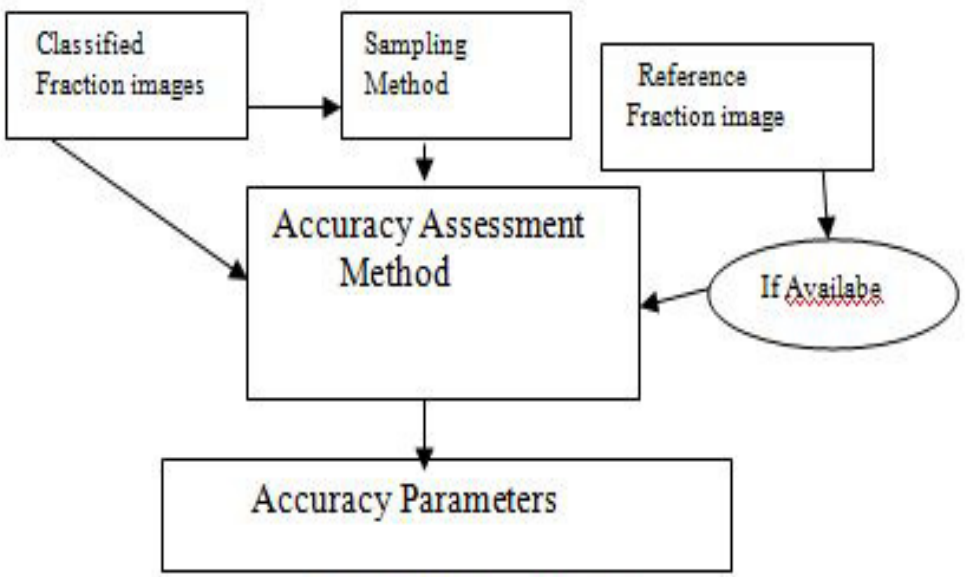

Fig.5: Methodology of research

Figure 4.1: Methodology of research

It has been recognized from the obtained results that irrespective of datasets $v=7 \times 10^{2}$ found more suitable to classify agriculture and Bright forest. However, for dry land, moist land and water body, $v=10^{2}$ is found to be more suitable for the classification using FCMWE classification approach. For Dense forest $v=4 \times 10^{2}$ is found to be more appropriate for classification. To perform classification a constant value of weighting exponent $\mathrm{m}=1$ has been used. 


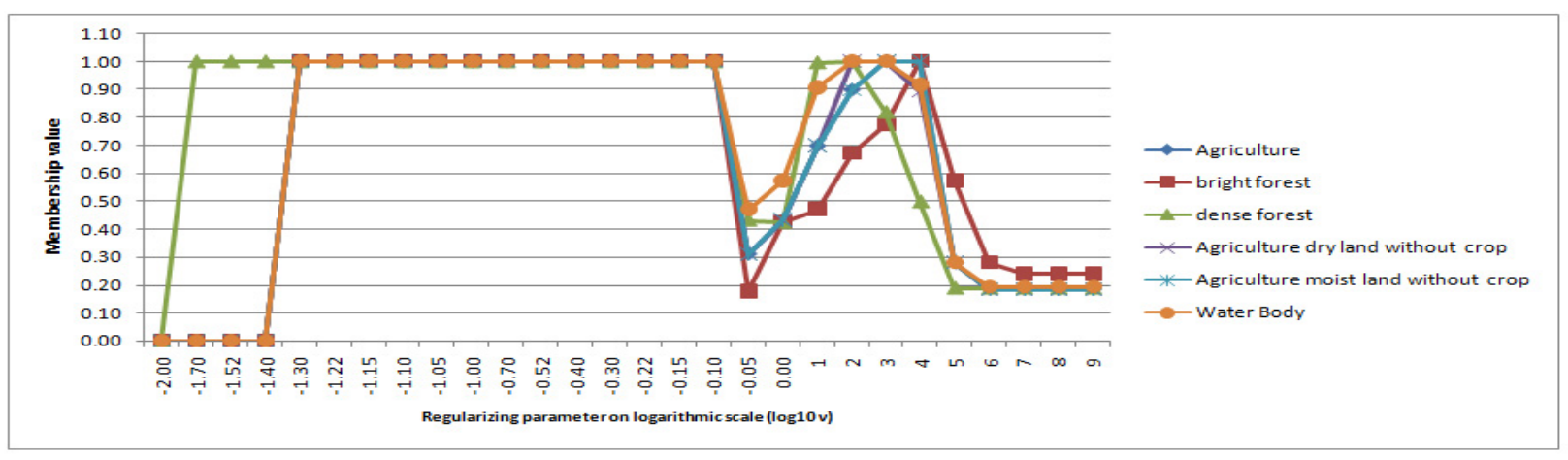

Figure 5.1: Class membership for FCMWE classifier using AWiFS datasets

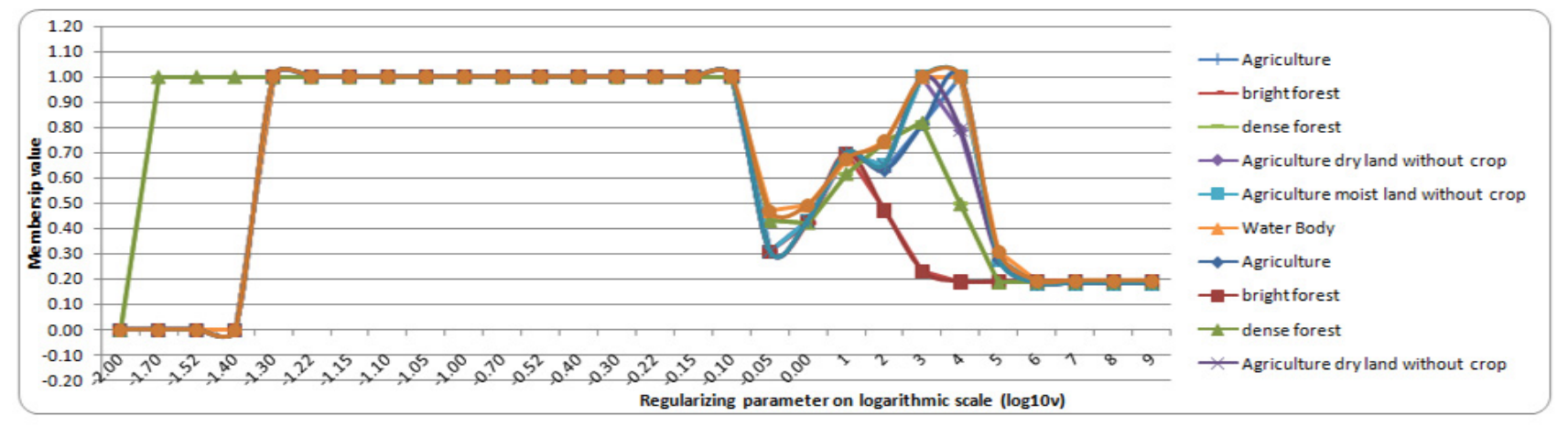

Figure 5.2: Class membership for FCMWE classifier using LISS- III datasets

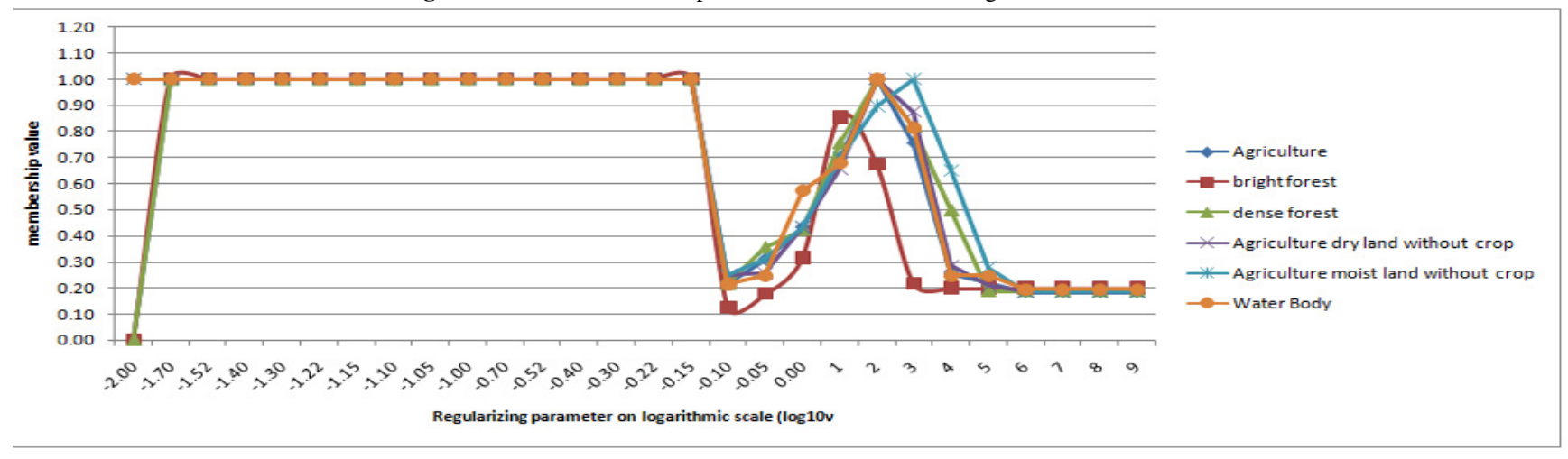

Figure 5.3: Class membership for FCMWE classifier using LISS- IV datasets

\section{Conclusion}

Fig. (6.1) shows the fraction images of AWiFS datasets for FCMWE classification. After examining the fraction images generated by FCMWE classifier, it has been observed that an intergrades phenomenon within pixel is more dominant in AWiFS imagery. It is shown in fraction images that regularizing parameter $v$ regularizes the output to remove inter-grade phenomena by using FCMWE classifier which removes uncertainty among classes. In FCMWE classifier the effect of regularizing parameter $(v)$ is dominant because of unity value of weighting exponent. This trend can be seen from fraction images \{Fig. (6.1) $\}$ where actual class produces high membership and all remaining classes are reflecting very low membership i.e. almost zero.

In this research paper, I have designed two hybrid soft classification algorithms. Adding Regularization parameter $(0<v<\infty)$ in these algorithms we are getting better classification with low entropy.

\section{References}

[1] M. K. Arora, A. Peterson, (Land cover classification from remote sensing data, GIS development6, 2002,24-25, 30-31.

[2] M. A.Aziz, Evaluation of soft classifiers for remote sensing data, Abstr. Appl. Anal.,unpublished Ph.D thesis, Indian Institute of Technology Roorkee,Roorkee,India. (2004).

[3] J.C.Bezdek, Pattern Recognition with Fuzzy Objective Function Algorithm,Plenum, New York, USA,(1981), ISBN: 978-1-4757-0452-5.

[4] J.C.Bezdek,R.Ehrlich,W.Full, The fuzzy c-means clustering algorithm, Computers and Geosciences, 1984,10,191-203.

[5] R. G.Congalton, A review of assessing the accuracy of classifications of remotely sensed data, (1991),37,35-47. 


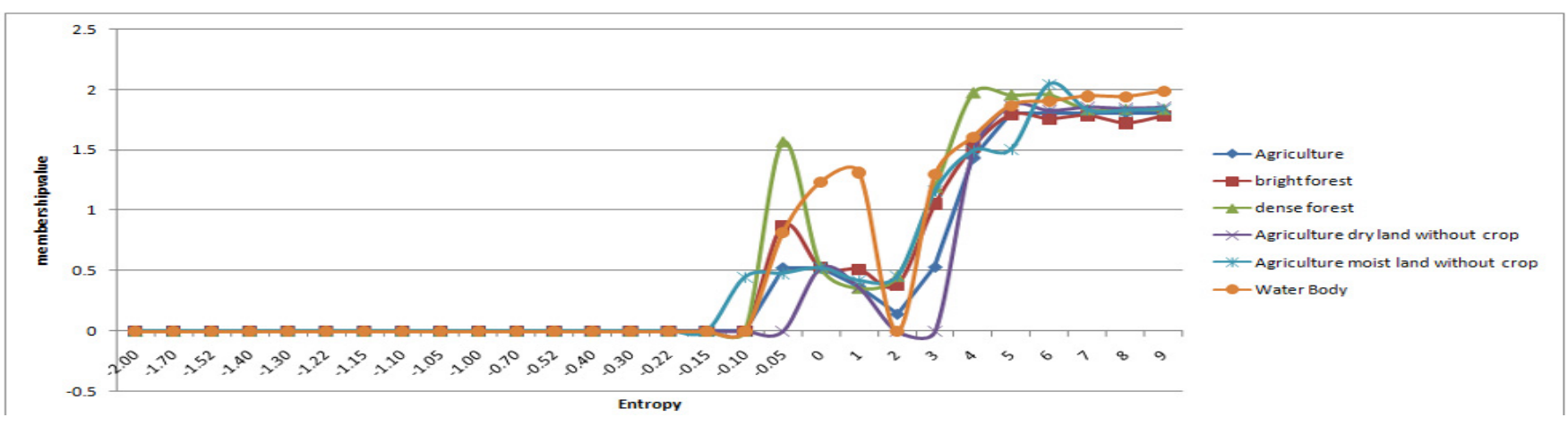

Figure 5.4: Entropy for FCMWE classifier using AWiFS dataset

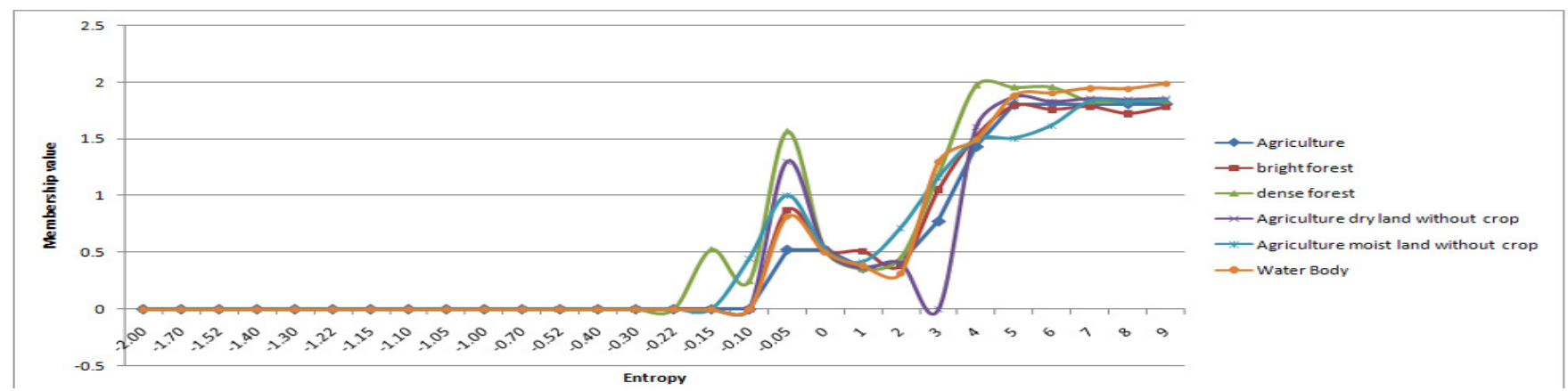

Figure 5.5: Entropy for FCMWE classifier using LISSIII dataset

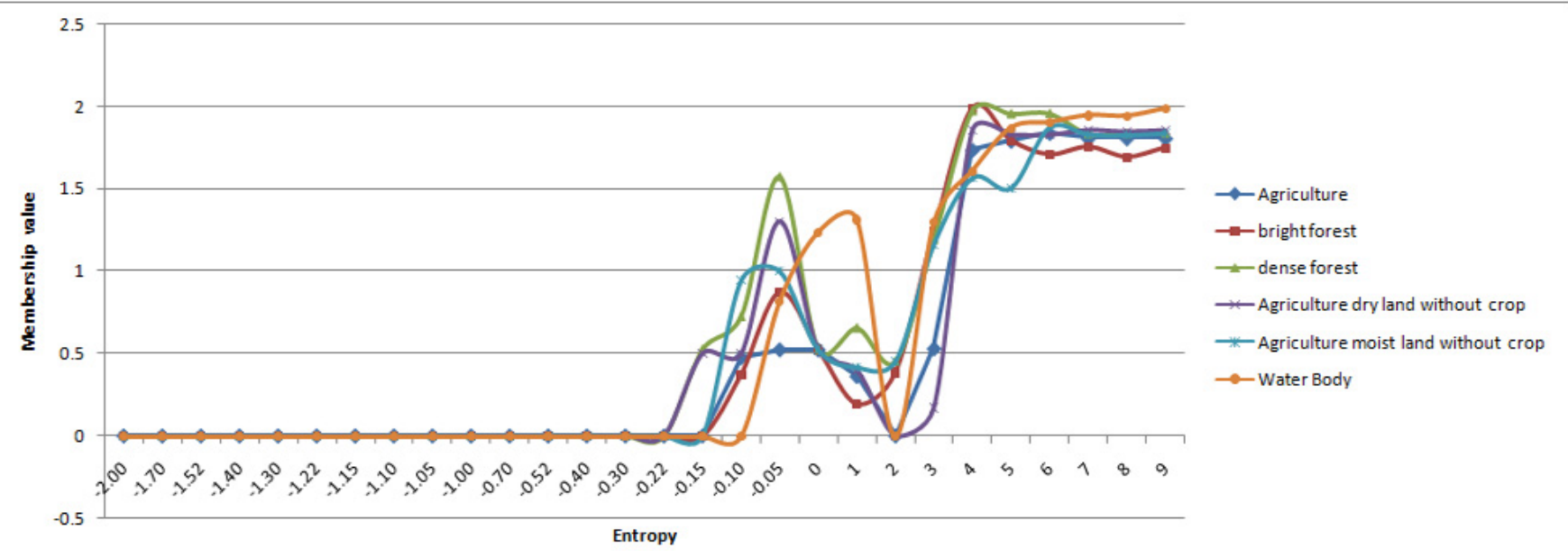

Figure 5.6: Entropy for FCMWE classifier using LISS-IV dataset

[6] H.Dehghan,H.Ghassemian, Measurement of uncertainty by the entropy: application to the classification of MSS data, International journal of remote sensing,(2006) vol.27, no. 18, 4005-4014.

[7] J.C.Dunn, Well Separated Clusters and Optimal Fuzzy Partitions, Journal of Cybernetics,(1981),4,95-104.

[8] R.k.Dwivedi,S. K.Ghosh, P.Roy, Optimization of Fuzzy Based Soft Classifiers for Remote Sensing Data,ISPRS-International Archives of the Photogrammetric, Remote Sensing and Spatial Information Sciences 1,(2012),385-390.

[9] R.k.Dwivedi,S. K.Ghosh, and Anil Kumar, Investigation Of Image Classification Techniques For Performance Enhancement,International Journal of Management and Technology, Volume 3, number 1, (2012),21-33.

[10] R.k.Dwivedi,S. K.Ghosh, Visualization of Uncertainty usingentropy on Noise clustering with entropy classifier, 3rd IEEE International Advance Computing Conference, (IACC-2013).(2013), ISBN:-978-1-4673-4528-6.

[11] J.R.Eastman, R.M.Laney, Bayesian soft classification for sub-pixel analysis: critical evaluation, Photogrammetric Engineering and Remote Sensing,68, (2002), 1149-1154.

[12] D.Ferna.Ndez-Prieto, An iterative approach to partially supervised classification problems, International Journal of Remote Sensing, 23,(2002), 3887-3892.

[13] G.M.Foody,Approaches for the production and evaluation of fuzzy land cover classifications from remotely-sensed data, Journal of Remote Sensing, vol.17, no. 7,(1996),1317-1340.

[14] G.M.Foody,Estimation of sub-pixel land cover composition in the presence of untrained classes, Computers and Geosciences, vol. 26, no. 4, (2000), 469-478.

[15] S. R.Kannan,R. Devi,S.Ramathilagam,K.Takezawa,Effective FCM noise clustering algorithms in medical images,Computers in Biology and Medicine,(2013), vol. 43, no.2, 73-83.

[16] J.Kivinen,M.Warmuth, Boosting as Entropy Projection,Proc. 12th Ann.Conf. Computational Learning Theory, (1999),134-144.

[17] R.Krishnapuram, J.M.Keller, A possiblistic approach to clustering,IEEE Transactions on Fuzzy Systems, 1,(1993), 98-108.

[18] A.Kumar, V. K.Dadhwal,Entropy based fuzzy classification parameter optimization using uncertainty variation across spatial resolution,Journal of Indian Society of Remote Sensing, (2010), Vol 38, No. 2,179-192.

[19] R.P.Li, M.Mukaidono, Gaussian clustering method based on maximum-fuzzyentropy interpretation,Fuzzy Sets and Systems 102,(1999) 253-258.

[20] D.Lu,Q. Weng, A survey of image classification methods and techniques for improving classification performance, International Journal of Remote Sensing, 28(5),(2007),823-870. 

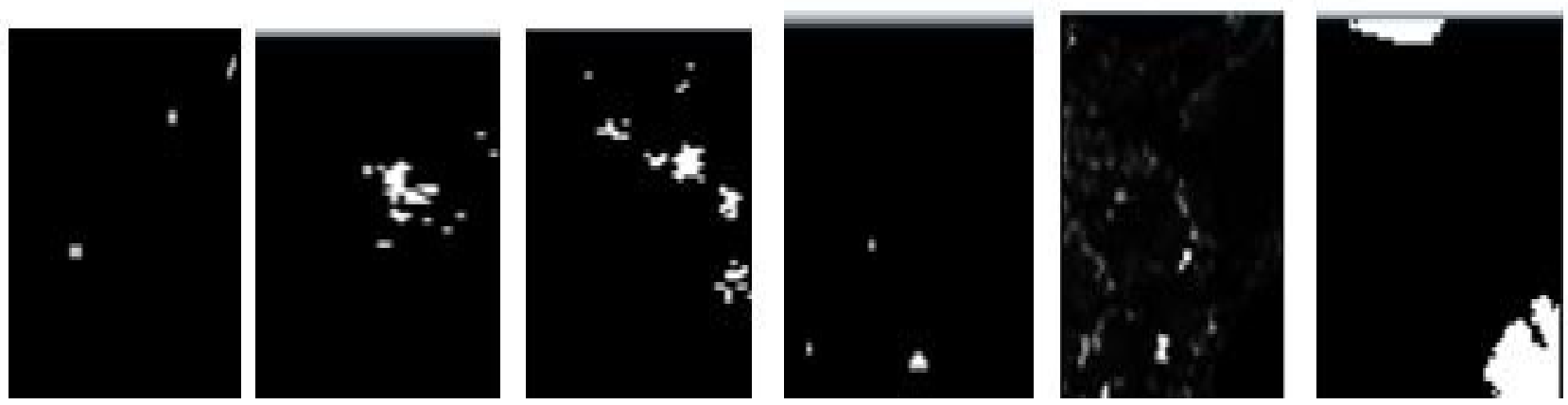

Figure 6.1: Entropy for FCMWE classifier using LISS-IV dataset

[21] A.M.Massone,F.Masulli,A.Petrosini, Fuzzy clustering algorithms and Landsat images for detection of waste areas: A comparison,In Advances in Fuzzy Systems and Intelligent Technologies,Proc. WILF '99, Italian Workshop on Fuzzy Logic,Shaker Publishing, Maastricht, The Netherlands,(2000), 165-175.

[22] S.Miyamoto,M.Mukaidono, Fuzzy c-means as a regularization and maximumentropy approach,In: Proc. of the 7th International Fuzzy Systems Association World Congress, Prague, Czech,(1997), June 25-30, 1997, vol. II, 86-92.

[23] K.Oki,T.M.Uenishi,K.Omasa, M.Tamura, Accuracy of land cover area estimation from coarse spatial resolution images using an unmixing method,International Journal of Remote Sensing, 25, (2004),1673-1683.

[24] I.Olthof, D.J.King, R.A.Lautenschlager, Mapping deciduous forest ice storm damage using Landsat and environmental data,Remote Sensing of Environment, 89, (2004),484-496.

[25] R.L.Powell,N.Matzke,C.De Souza JR,M.Clark,I.Numata,L.L.Hess, D.A.Roberts, Sources of error in accuracy assessment of thematic land-cover maps in the Brazilian Amazon,Remote Sensing of Environment, 90, (2004),221-234.

[26] Priyadarshi Upadhyay, S. K. Ghosh, and Anil Kumar, A Brief Review of Fuzzy Soft Classification and Assessment of Accuracy Methods for Identification of Single Land Cover,Studies in Surveying and Mapping Science (SSMS) American Society of Science and Engineering, Volume 2, (2014),ISSN 2328-6245.

[27] R.k.Dwivedi,S. K.Ghosh,A.Kumar, Investigation Of Image Classification Techniques For Performance Enhancement, Viewpoint "An International Journal of Management and Technology",(2012),ISSN-2229-3825.

[28] Ranjana Sharma,Achal Kumar Goyal,Achal Kumar Goyal, A Review of Soft Classification Approaches on Satellite Image and Accuracy Assessment, Proceedings of Fifth International Conference on Soft Computing for Problem Solving, Springer Singapore, (2016),vol.437,629-639.

[29] S. S.Sengar,A.Kumar,H. R.Wason,S. K.Ghosh,Krishna Murthy,sh, Study of soft classification approaches for identification of earthquake-induced liquefied soil,Geomatics, Natural Hazards and Risk(ahead-of-print), (2013),1-19.

[30] M.A.Shalan,M.K.Arora,S.K.Ghosh, An evaluation of fuzzy classifications from IRS IC LISS III imagery: a case study, International Journal of Remote Sensing,24,(2003), 3179-3186.

[31] C. E.Shannon, A Mathematical Theory of Communication,Bell System Technical Journal 27(3), (1948),379-423.

[32] C. E.Shannon, Prediction and Entropy of Printed English,Bell System Technical Journal 30 (1), (1951),50-64.

[33] Xiao-Hong Wu,Jian-Jiang Zhou, Alternative Noise Clustering Algorithm,IEEE- ICSP Proceedings, (2006),0-7803-9737-1/06.

[34] A.N.Tihonov,V.Y.Arsenin, Solutions of Ill-Posed Problems,. Wiley, New York,(1977).

[35] V.Vapnik, The Nature of Statistical Learning Theory,Springer Verlag, New York,(1995).

[36] V.Vapnik, IStatistical Learning Theory, John Wiley and Sons,New York,(1998).

[37] V. N.Vapnik, An overview of statistical learning theory,IEEE Transactions of Neural Networks, 10, (1999),988-999.

[38] V. N.Vapnik, The Nature of Statistical Learning Theory,2nd Edition,(2000).

[39] J.Zhang,G.M.Foody, A fuzzy classification of sub-urban land cover from remotely sensed imagery,International Journal of Remote Sensing, 19,(2001), $2721-2738$ 